\title{
Telomere und Telomerase als Ansatzpunkte neuer Therapieformen in der Onkologie
}

Redaktion: H.E. Blum, Freiburg

Ein wesentliches Merkmal von Tumorzellen ist ihr unbegrenztes Wachstumspotenzial. Im Gegensatz dazu steht die begrenzte Zellteilungsfähigkeit (ca. 4060x) normaler somatischer Zellen. Der Mechanismus, der die Anzahl der Zellteilungen festlegt, ist an die funktionale Integrität der Chromosomenenden, der so genannten Telomere, gebunden. Tumorzellen können die Funktion ihrer Telomere durch Expression des Enzyms Telomerase aufrechterhalten, so dass sich daraus ein neuer therapeutischer Ansatzpunkt für die Tumorbehandlung ergibt.

\section{Telomere}

Telomere bestehen aus sich tausendfach wiederholenden, nicht kodierenden $\mathrm{T}_{2} \mathrm{AG}_{3}$-Sequenzen, die durch spezifische telomerbindende Proteine eine Lasso- oder Loop-Formation einnehmen $(1,2)$. Durch diese besondere Architektur liegen die Chromosomenenden nicht frei vor, sondern die Telomere bilden eine Art molekularer Kappen, die die Chromosomen vor Prozessen wie Degradierung, Rekombination und Fusionen schützen. Jedoch besteht gerade an den Enden der Chromosomen ein besonderes Problem bei der Replikation der DNA, das bereits bei der Entschlüsselung der Doppelhelixstruktur als End-Replikationsproblem beschrieben wurde: Mit jeder Zellteilung geht ein geringer Teil der endständigen DNA verloren (Abb.1). Der daraus resultierende Verlust von Telomersequenzen führt daher ab einer kritischen Schwelle zu einer Aufhebung der Loop-Struktur (= dysfunktionelle Telomere), wodurch die protektive Funktion der Telomere entfällt. Als Konsequenz werden die Chromsomen instabil, gehen verloren oder verbinden sich untereinander. Eine Akkumulation solcher DNA-Schäden kann zu einem Zellwachstumsstopp oder zu Apoptose führen. Demnach ist die "biologische Uhr", die die Anzahl der Zellteilungen bestimmt, an

Dtsch Med Wochenschr 2001; 126: 1171-1172 (c) Georg Thieme Verlag Stuttgart · New York

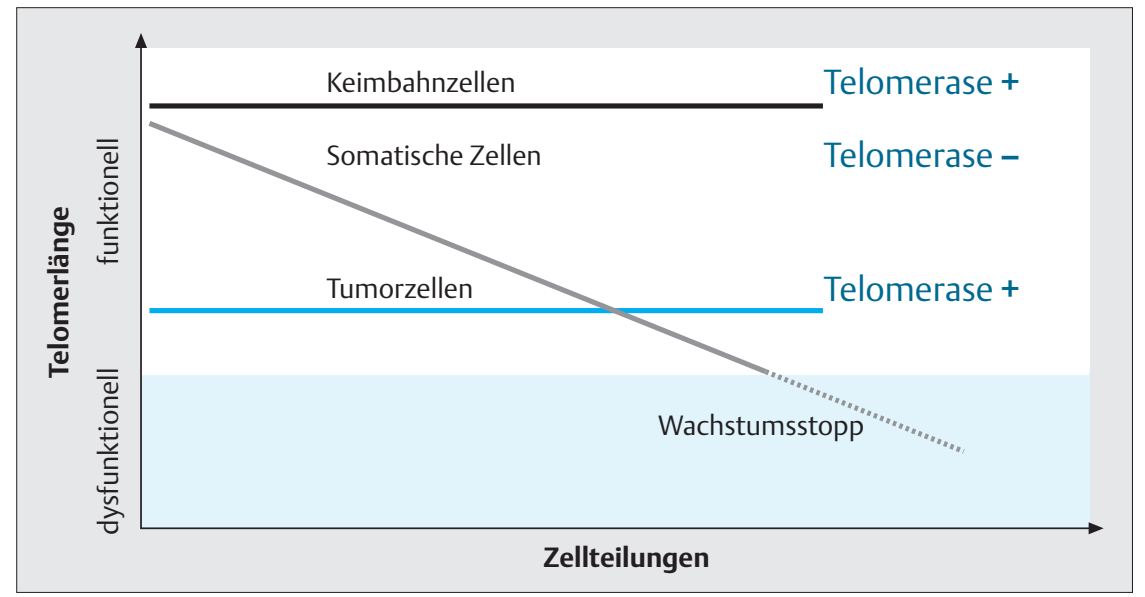

Abb.1 Telomerlängendynamik unterschiedlicher Gewebe: Normale somatische Zellen haben in der Regel keine Telomerase-Aktivität. Ihre Zellteilungsfähigkeit ist durch den progredienten Telomerverlust limitiert. Keimbahnzellen haben lange Telomere, die durch die hohe Telomerase-Aktivität konstant gehalten werden. Tumorzellen haben meistens kurze Telomere. Ihre Zellteilungsfähigkeit ist uneingeschränkt, da sie durch die vorhandene Telomerase-Aktivität ihre Telomere erhalten können.

die Länge und funktionale Integrität der Telomere gebunden.

\section{Telomerase}

Im Gegensatz zu somatischen Zellen müssen Keimbahnzellen den Telomerverlust kompensieren können, da eine Begrenzung ihrer Lebensspanne nicht mit Fortpflanzung vereinbar wäre. Sie lösen dieses spezielle Problem durch die reverse Transkriptase Telomerase (Abb.1). Dieses Enzym besteht aus mehreren Proteineinheiten und einer RNA-Einheit, die als Matrize für die Neusynthese von $\mathrm{T}_{2} \mathrm{AG}_{3}$-Sequenzen dient. Die wichtigste und aktivitätslimitierende Komponente ist dabei die katalytische Untereinheit hTERT (humane reverse Transkriptase). Im Vergleich zu Keimbahnzellen ist die Expression von Telomerase in somatischen Zellen in der Regel unterdrückt. Tumorzellen dagegen können im Laufe der malignen Transformation Telomerase reaktivieren, wodurch die Telomere stabilisiert werden und der Verlust von $\mathrm{T}_{2} \mathrm{AG}_{3}$-Sequenzen kompensiert werden kann. Die Folge ist die Fähigkeit des Tumorklons zur unbegrenzten Proliferati- on. Umfangreiche Studien konnten belegen, dass ca. 85\% aller primären $\mathrm{Tu}$ more im Gegensatz zu umgebendem normalem Gewebe hohe Telomeraseaktivität mittels eines PCR-basierten Assays (TRAP-Assay) zeigen (3). Darüber hinaus ist die Induktion von Telomerase neben der Aktivierung der Onkogene ras und SV40 large T antigen als einer von drei essentiellen Schritten anzusehen, um im einem In-vitro-Modellsystem aus einer normalen Fibroblastenoder Epithelzelle eine Tumorzelle zu generieren.

\section{Telomerase-Hemmung}

Viel Aufmerksamkeit ist gegenwärtig auf die Frage gerichtet, ob die spezifische Hemmung der Telomerase einen neuen Ansatz in der Tumortherapie darstellen könnte. Der Beweis für die Wirksamkeit der Telomerasehemmung konnte in zwei grundlegenden Arbeiten demonstriert werden, bei denen eine Inhibition des Enzyms an humanen Tumorzellen mittels genetischer Methoden vorgenommen wurde $(4,5)$. Dabei konnte durch Einführung eines mutierten hTERT-Gens in mehrere Tumor-Zelllini- 


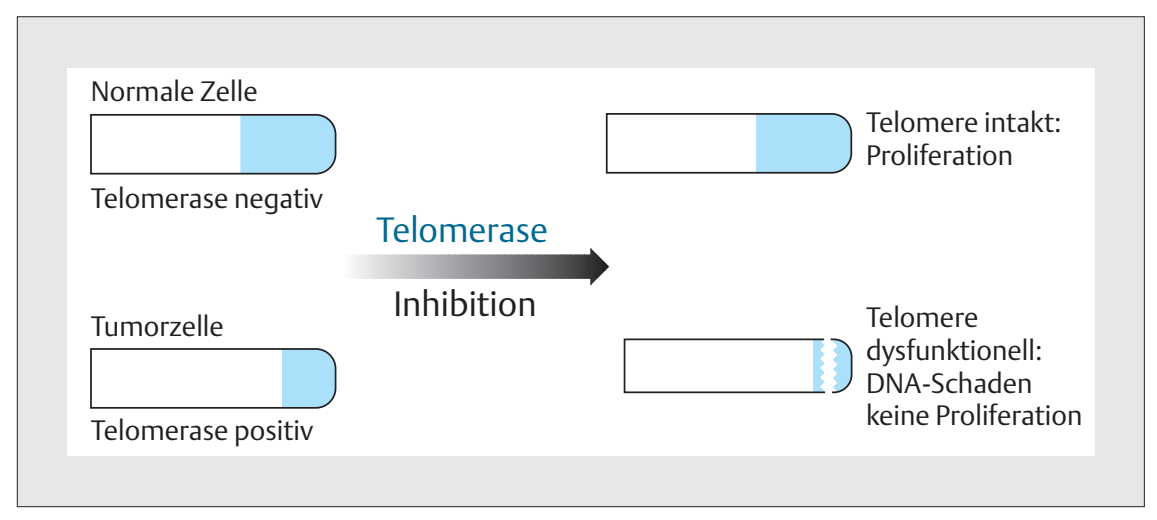

Abb.2 Modell des Einflusses der Telomerase-Hemmung auf das Wachstum von normalen und malignen Zellen: Normale Zellen ohne Telomerase-Aktivität bleiben unbeeinflusst. Die kurzen Telomere der Tumorzellen werden ohne Telomerase-Aktivität nach einer Latenzzeit dysfunktionell und führen zu einem Wachstumsstopp bzw. zu Apoptose.

en die Telomeraseaktivität komplett unterdrückt werden. Als Konsequenz verkürzten sich die Telomere dieser Zellen und lösten nach einem variablen Zeitintervall ein Seneszenz- oder Apoptoseprogramm aus. Die Latenzzeit des antiproliferativen Effektes war dabei abhängig von der Ausgangs-Telomerlänge der untersuchten Gewebe. Das heißt, dass Tumorzellen mit kurzen Telomeren nach Telomerasehemmung schneller dysfunktionell werden als solche mit langen Telomeren. Demnach ist nicht die Telomerase-Inhibition per se für den antiproliferativen Effekt verantwortlich, sondern der fehlende Telomererhalt mit konsekutivem Erreichen einer kritischen Telomerlänge (Abb.2). Beeindruckend ist ferner die Beobachtung, dass die humanen Tumorzelllinien, bei denen Telomerase-Aktivität unterdrückt ist, die Fähigkeit verlieren, Tumoren in immundefizienten Mäusen auszubilden. Dabei scheint nach gegenwärtigem Kenntnisstand bei humanen Tumoren ein antiproliferativer Effekt unabhängig vom Status des Tumorsuppressorgens p53 zu sein.

Gegenwärtig wird intensiv an der Entwicklung spezifischer Inhibitoren auf pharmakologischer Basis (small molecules) gearbeitet. Zwar sind bereits einige Wirkstoffe in der Literatur beschrieben worden (6), wobei jedoch kritisch angemerkt werden muss, dass deren Potenz im Hinblick auf eine spezifische Telomerase-Inhibition zu gering (hohe $\mathrm{IC}_{50}$-Werte) und daher eine Anwendung in der klinischen Situation nicht aussichtsreich erscheint. Darüber hinaus konnte bei diesen Substanzen in den präklinischen Modellen keine aus- reichende Telomerverkürzung induziert werden, was jedoch im Hinblick auf die Untersuchungen mit genetisch induzierten Telomerase-Inhibition essenziell ist (siehe oben). Dennoch ist zu erwarten, dass in nächster Zukunft verbesserte, spezifischere Wirkstoffe identifiziert werden, die den Einzug in klinische Studien erhalten werden. Grundsätzlich ist bei der Anwendung von Telomerase-Inhibitoren in der klinischen Situation zu berücksichtigen, dass ein antiproliferativer Effekt von der jeweiligen Länge der Telomere abhängt, so dass kein unmittelbarer, sondern ein verzögerter therapeutischer Effekt zu erwarten ist. Dies bedeutet, dass die Tumorlast eher gering sein sollte, da unter der Inhibitortherapie zunächst eine Latenzzeit mit weiterem Tumorprogress resultieren würde, bis schließlich ein antiproliferativer Effekt eintritt. Der Einsatz von Telomerase-Inhibitoren wäre daher in erster Linie additiv zu anderen zytoreduktiven Therapien denkbar. Eine potenzielle Indikation für die Monotherapie mit Telomerase-Inhibitoren stellt die Eradikation einer minimalen residualen Erkrankung (MRD) als Postremissionstherapie dar sowie möglicherweise die Chemoprävention bei prämalignen Erkrankungen.

Trotz der faszinierenden Entwicklungen auf dem Gebiet der Grundlagenforschung sollte nicht unberücksichtigt bleiben, dass bei ca. $10 \%$ primärer Tumoren so genannte alternative Telomer-Verlängerungsmechanismen beschrieben worden sind, die man ALT ( $=$ alternative lengthening of telomeres) nennt und die potenziell einen Resistenzmechanismus darstellen könnten.
Allerdings muss im Hinblick auf einen therapeutischen Einsatz dagegengehalten werden, dass ALT bisher nicht bei der genetischen Telomerase-Inhibition von Tumorzellinien und am Mausmodell beobachtet worden sind. Jedoch ist bei der 293-Zelllinie (embryonale Nierenzelllinie) in zwei Klonen eine Reaktivierung von Telomerase beobachtet worden, deren Ursache unklar ist und möglicherweise eine Genamplifikation von hTERT widerspiegelt (5). Weiterhin ist zu berücksichtigen, dass Telomerase-Aktivität in geringem Ausmaß auch in Zellen mit selbsterneuerndem Potenzial wie Stammzellen oder Lymphozyten nachweisbar ist, so dass ein Einsatz von Telomerase-Inhibitoren theoretisch auch zu einem akzelerierten Telomerverlust in diesen Zellen beitragen kann. Ob dadurch eine relevante Proliferationshemmung mit einhergehender Stammzell- oder Immundysfunktion resultieren kann, ist eher unwahrscheinlich, da diese Zellen in der Regel lange Telomere haben. Allerdings gibt es zu dieser Frage bislang keine experimentellen Daten.

Die präferenzielle Expression von Telomerase ermöglicht die Entwicklung tumorspezifischer Wirkstoffe mit geringer Toxizität. Die Wirksamkeit und der Stellenwert dieser Substanzen für die Tumortherapie muss schließlich im Rahmen klinischer Studien evaluiert werden.

\section{Literatur}

1 Hoos A, Nekarda H. Telomerase - Potential und Grenzen der klinischen Anwendbarkeit. Dtsch Med Wochenschr 1999; 124: 223-230

2 Collins K. Mammalian telomeres and telomerase. Curr Opin Cell Biol 2000; 12: 378383

3 Shay JW, Bacchetti S. A survey of telomerase activity in human cancer. Eur J Cancer 1997; 33: 787-791

4 Hahn WC et al. Inhibition of telomerase limits the growth of human cancer cells. Nat Med 1999; 5: 1164-1170

5 Zhang $\mathrm{X}$ et al. Telomere shortening and apoptosis in telomerase-inhibited human tumor cells. Genes Dev 1999; 13: 2388-2399

6 White LK, Wright WE, Shay JW. Telomerase inhibitors. Trends Biotechnol 2001; 19: 114120

Dr. med. Uwe M. Martens

Priv.-Doz. Dr. med.

Cornelius F. Waller

Medizinische Universitätsklinik

Abteilung Hämatologie/Onkologie

Hugstetterstraße 55

79106 Freiburg i. Br.

E-Mail: martens@ukl.uni-freiburg.de 